\title{
A detailed clinical and molecular survey of subjects with nonsyndromic USH2A retinopathy reveals an allelic hierarchy of disease-causing variants
}

\author{
Eva Lenassi ${ }^{1,2}$, Ajoy Vincent ${ }^{3}$, Zheng $\mathrm{Li}^{1,4}$, Zubin Saihan ${ }^{1}$, Alison J Coffey ${ }^{5}$, Heather B Steele-Stallard ${ }^{6}$, \\ Anthony T Moore ${ }^{1}$, Karen P Steel ${ }^{5}$, Linda M Luxon ${ }^{7,8}$, Elise Héon ${ }^{3}$, Maria Bitner-Glindzicz ${ }^{6}$ \\ and Andrew R Webster ${ }^{\star}, 1$
}

\begin{abstract}
Defects in USH2A cause both isolated retinal disease and Usher syndrome (ie, retinal disease and deafness). To gain insights into isolated/nonsyndromic USH2A retinopathy, we screened USH2A in 186 probands with recessive retinal disease and no hearing complaint in childhood (discovery cohort) and in 84 probands with recessive retinal disease (replication cohort). Detailed phenotyping, including retinal imaging and audiological assessment, was performed in individuals with two likely disease-causing USH2A variants. Further genetic testing, including screening for a deep-intronic disease-causing variant and large deletions/duplications, was performed in those with one likely disease-causing change. Overall, 23 of 186 probands (discovery cohort) were found to harbour two likely disease-causing variants in USH2A. Some of these variants were predominantly associated with nonsyndromic retinal degeneration ('retinal disease-specific'); these included the common c.2276 G > T, p.(Cys759Phe) mutation and five additional variants: c.2802 T > G, p.(Cys934Trp); c.10073 G >A, p. (Cys3358Tyr); c.11156 G >A, p.(Arg3719His); c.12295-3 T $>$ A; and c.12575 G >A, p.(Arg4192His). An allelic hierarchy was observed in the discovery cohort and confirmed in the replication cohort. In nonsyndromic USH2A disease, retinopathy was consistent with retinitis pigmentosa and the audiological phenotype was variable. USH2A retinopathy is a common cause of nonsyndromic recessive retinal degeneration and has a different mutational spectrum to that observed in Usher syndrome. The following model is proposed: the presence of at least one 'retinal disease-specific' USH2A allele in a patient with USH2A-related disease results in the preservation of normal hearing. Careful genotype-phenotype studies such as this will become increasingly important, especially now that high-throughput sequencing is widely used in the clinical setting.
\end{abstract}

European Journal of Human Genetics (2015) 23, 1318-1327; doi:10.1038/ejhg.2014.283; published online 4 February 2015

\section{INTRODUCTION}

Retinitis pigmentosa is the most common inherited retinal degeneration and a major cause of visual impairment among individuals aged $20-64$ years. $^{1,2}$ It is genetically heterogeneous (over 60 genes implicated so far) and associated with significant variability in age of onset, disease progression and retinal appearance (RetNet; http://www.sph. uth.tmc.edu/retnet/, accessed 31 December 2014). ${ }^{3}$ Although retinitis pigmentosa is a disease confined to the eye, some $20-30 \%$ of patients have associated non-ocular disease; Usher syndrome, in which retinitis pigmentosa is combined with, typically prelingual, sensorineural hearing loss, is the most frequent syndromic form. ${ }^{4}$

Disease-causing variants in the USH $2 A$ gene are the most common cause of Usher syndrome (29\% of all cases) and one of the most common causes of nonsyndromic autosomal recessive retinitis pigmentosa (19-23\% of all cases). ${ }^{4,5}$ The USH2A gene is located on 1q41 and has two alternatively spliced transcripts: a short one consisting of 21 exons, and a longer one consisting of 51 additional 3' exons; the latter encodes a 5202 amino-acid matrix protein expressed specifically in photoreceptors and developing cochlear hair cells. ${ }^{6,7}$ It has been shown that the USH2A protein is required for long-term maintenance of retinal photoreceptors and the development of cochlear cells. ${ }^{7}$

Over 2900 coding variants have been reported in the USH2A gene (1000 genomes project database, National Heart, Lung and Blood Institute Exome Sequencing Project or NHLBI ESP and LOVDUSHBase, accessed 15 September 2014). Over 470 of these changes are presumed to be pathogenic (HGMD, accessed 15 September 2014); most of these disease-causing variants are found in one or a few cases each, with the exception of c.2299delG, p.(Glu767Serfs $\left.{ }^{\star} 21\right)$ and c.2276 G > T, p.(Cys759Phe), which are more commonly found in patient cohorts. The c.2299delG variant causes a substantial proportion of cases of Usher syndrome, ${ }^{8}$ while the c.2276 G>T change has been associated mainly with disease confined to the eye. ${ }^{9,10}$ This suggests the existence of alleles that are likely to be specific to those with nonsyndromic disease ('retinal disease-specific'). However, this has not been studied in detail and to date no other such 'retinal disease-specific' alleles have been identified.

Here, we have performed a comprehensive screen of the USH2A gene in 186 patients with autosomal recessive retinal degeneration and

${ }^{1}$ UCL Institute of Ophthalmology and Moorfields Eye Hospital, University College of London, London, UK; ${ }^{2}$ Eye Hospital, University Medical Centre, Ljubljana, Slovenia; ${ }^{3}$ The Hospital for Sick Children, Department of Ophthalmology and Vision Sciences, University of Toronto, Toronto, ON, Canada; ${ }^{4}$ Ocular Genetics, Singapore Eye Research Institute, Singapore, Singapore; ${ }^{5}$ Wellcome Trust Sanger Institute, Hinxton, UK; ${ }^{6}$ UCL Institute of Child Health, London, UK; ${ }^{7}$ UCL Ear Institute, London, UK; ${ }^{8}$ National Hospital for Neurology and Neurosurgery, London, UK

*Correspondence: Professor AR Webster, UCL Institute of Ophthalmology and Moorfields Eye Hospital, University College of London, 11-43 Bath Street, London EC1V 9EL, UK. Tel: +44 207566 2260; Fax: +44 207608 6830; E-mail: andrew.webster@ucl.ac.uk

Received 18 June 2014; revised 20 November 2014; accepted 2 December 2014; published online 4 February 2015 
no complaint of childhood hearing loss to gain insights into nonsyndromic USH2A-related disease. Detailed phenotyping, including fundus autofluorescence imaging and audiological assessment, was performed in individuals found to harbour two likely disease-causing variants. The effect of three splice site changes on nasal mRNA was investigated and multiplex ligation-dependent probe amplification (MLPA) was performed in selected patients with the aim of detecting deletions and duplications in USH2A. Additionally, we have investigated whether nonsyndromic versus syndromic disease can be explained by the presence of an allelic hierarchy of USH2A diseasecausing variants, and have addressed this by studying USH $2 A$ allelic heterogeneity in the discovery $(n=186)$ and two additional $(n=84$ and $n=187)$ cohorts.

\section{MATERIALS AND METHODS}

Study subjects

A total of 186 unrelated individuals with recessive retinal degeneration and no hearing complaint in childhood were ascertained from the clinics of Moorfields Eye Hospital (London, UK; discovery cohort). Of these, 168 patients were affected with rod-cone dystrophy (ie, retinitis pigmentosa), 12 with cone-rod dystrophy and 6 with childhood-onset retinal dystrophy. Further to this discovery cohort, 84 additional probands with recessive retinal degeneration (62 with nonsyndromic disease and 22 with Usher syndrome) were recruited at the Hospital for Sick Children (Toronto, ON, Canada); both cohorts underwent full sequencing of USH2A (see below). A third cohort of 187 unrelated patients with nonsyndromic, adult-onset, retinitis pigmentosa was also ascertained from the Moorfields Eye Hospital. This cohort was only used to test for selected variants in exons 13,51, 57 and 63 . A complete eye examination was performed and a detailed clinical history was obtained in all patients. Only patients with a family history compatible with autosomal recessive inheritance (ie, no evidence of dominant inheritance) were studied. For the purposes of this study, we define the phenotype observed in retinitis pigmentosa patients without prelingual/childhood-onset hearing loss as nonsyndromic disease.
Subjects with Usher syndrome were not tested for common genetic causes of nonsyndromic hearing impairment.

After informed consent was obtained, blood samples were collected and genomic DNA was extracted from peripheral blood leucocytes. Control DNA and mRNA were obtained from consented unrelated healthy individuals. All investigations were conducted in accordance with the Declaration of Helsinki principles. Institutional Review Board (IRB)/Ethics Committee approval was obtained from the Moorfields Eye Hospital and the Hospital for Sick Children local ethics committees.

USH $2 A$ screening and method used to distinguish disease-causing variants

The 186 probands with recessive retinal degeneration and no hearing complaint in childhood and the 84 probands with recessive retinal degeneration underwent bidirectional DNA sequencing of the 72 exons of the USH $2 A$ gene. Primers were designed for all exons and intron-exon boundaries of the transcript with accession number NM_206933.2 (a total of 105 primer pairs; genomic reference sequence NG_008212.3). ${ }^{8}$ The additional panel of 187 unrelated individuals with nonsyndromic, adult-onset, recessive retinitis pigmentosa underwent bidirectional DNA sequencing of exons 13,51, 57 and 63 of the USH2A gene; these exons were the location of presumed 'retinal disease-specific' variants. In 24 patients from the discovery cohort, only one likely disease-causing $\mathrm{USH} 2 \mathrm{~A}$ variant was identified. These patients were: (i) sequenced for the USH2A c.7595-2144 A>G, p.Lys2532Thrfs ${ }^{*} 56$ change, which causes inclusion of a pseudoexon; ${ }^{11}$ and (ii) screened using MLPA to detect deletions and duplications in the USH2A gene. The SALSA MLPA FAMlabelled reagent kit with probe mixes P361-A1/P362-A2 developed by MRCHolland (MRC-Holland, Amsterdam, The Netherlands) was used and reactions were performed according to the manufacturer's instructions. Two subjects with previously identified heterozygous deletions were included to act as positive controls and confirm the validity of the method.

Sequence alterations were classified as 'likely disease-causing variants' if they (i) were either nonsynonymous (including missense) or coding insertions/ deletions or splice site (positions \pm 3 ) or large duplications/deletions and (ii) have a minor allele frequency (MAF) of $0.15 \%$ or less in the NHLBI ESP data
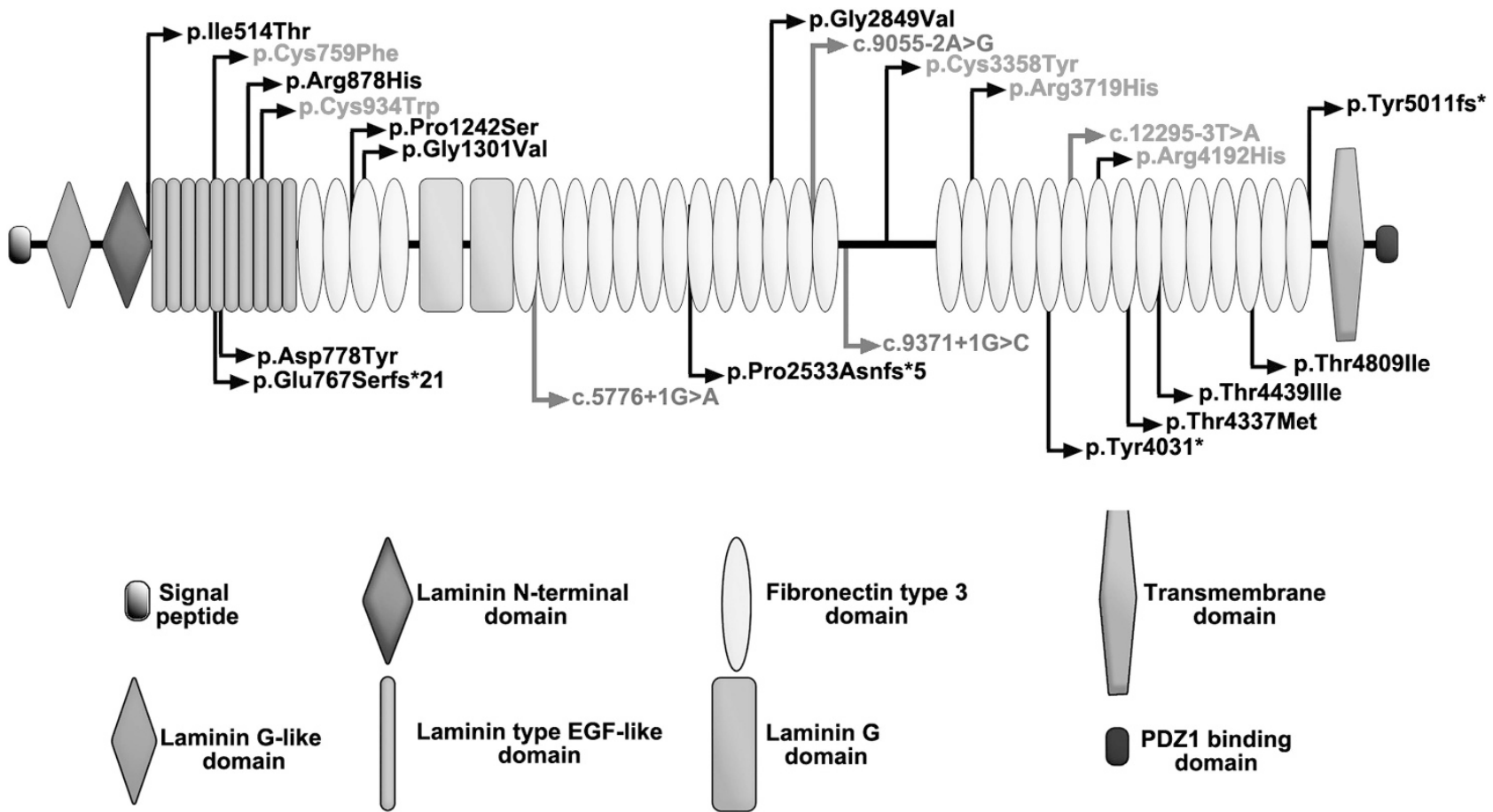

Figure 1 Schematic of the usherin protein and localisation of the likely disease-causing variants detected in the discovery cohort of patients ( $n=186$ ); mutations previously reported in individuals with Usher syndrome type II are shown below the schematic. Presumed 'retinal disease disease-specific' alleles are shown in red. 


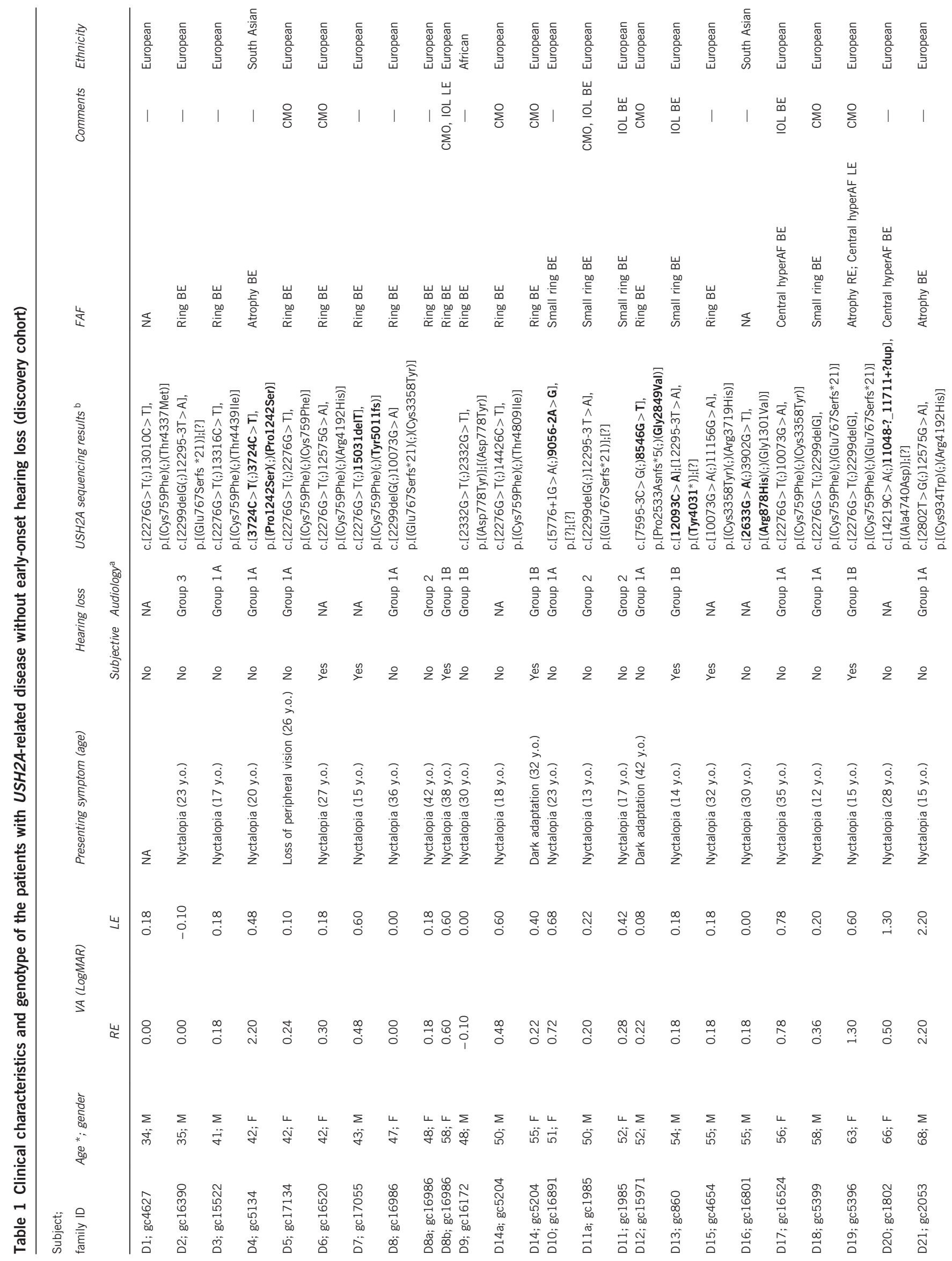


set (accessed 15 June 2014); this is the MAF of c.2276 G > T, the most common change identified in patients with recessive retinitis pigmentosa. ${ }^{9}$ The effect of synonymous variants on splicing was not assessed in the present study. All relevant data (variants and associated phenotypes) were submitted to the appropriate LOVD-USHBase, which can be accessed in http://www.LOVD.nl/ USH2A (submission IDs: $0004401-0004452$ ).

\section{Nasal epithelial mRNA analysis}

Nasal epithelial mRNA analysis was performed in individuals carrying the variants c.12295-3 T > A (subject D13), c.9056-2 A > G (subject D10) or c.5776 $+1 \mathrm{G}>\mathrm{A}$ (subject D10). Nasal epithelial cell samples were obtained by gently brushing the lateral inferior turbinate with bronchial cytology brush (Diagmed Ltd, North Yorkshire, UK) and RNA was extracted from these samples using the NucleoSpin RNA II Extraction Kit (Macherey-Nagel, Duren, Germany) according to the manufacturer's guidelines. cDNA was reverse transcribed using a cDNA Synthesis Kit (BioLine, London, UK) with a random hexamer primer mix. For reverse transcriptase (RT)-PCR reactions, USH2A was amplified between exons 27 and 32, exons 45 and 49, exons 58 and 64 and exons 62 and 66. The housekeeping gene $\beta$-actin was amplified as a positive control. The identity of USH2A RT-PCR products was established by direct sequencing using standard procedures (primer sequences and conditions are available on request).

\section{Ophthalmological and audiological assessment}

Detailed ophthalmological evaluation was performed in 23 probands with two likely disease-causing variants in USH2A (all from the discovery cohort); 4 affected siblings of the 23 probands were also assessed. Ophthalmological examination included best-corrected visual acuity testing, dilated fundus examination, colour fundus photography (TRC-50IA; Topcon, Tokyo, Japan), spectral domain optical coherence tomography (OCT) and fundus autofluorescence imaging. The Spectralis HRA+OCT with viewing module version 5.1.2.0 (Heidelberg Engineering, Heidelberg, Germany) was used to acquire tomographs in 24 patients; fundus autofluorescence images were acquired in 24 patients using the HRA2 and Spectralis HRA+OCT (over a $30^{\circ} \times 30^{\circ}$ and/or a $55^{\circ} \times 55^{\circ}$ field; Heidelberg Engineering) instruments.

Pure tone audiometry was conducted, in a sound-treated booth, using a calibrated GSI 61 audiometer with TDH 49 headphones to assess hearing thresholds ${ }^{12}$ in 19 patients with no complaint of hearing loss, who were found to have two disease-associated variants in USH2A (all from the discovery cohort); the method has been described previously. ${ }^{8}$ The audiology thresholds $(0.25-8 \mathrm{kHz})$ were reviewed for right and left ear separately and compared with age- and gender-matched percentile bands of normative data. ${ }^{13}$ Patients were classified into three groups: Group 1 with normal hearing thresholds falling in the interquartile normative range for age and gender across all frequencies $(1 \mathrm{~A}$ if all thresholds < 40th percentile; 1B if thresholds fell in the 50-60th percentile band); Group 2 with high-frequency hearing thresholds $(3-8 \mathrm{kHz})>75$ th percentile and markedly greater than low-frequency threshold $(0.25-2 \mathrm{kHz})$ percentiles (ie, 30-70 percentile bands difference); and Group 3 with atypical/ abnormal audiometric configurations ${ }^{14}$ and without other aetiological explanation.

\section{RESULTS}

$U S H 2 A$ retinopathy is a major cause of adult-onset recessive retinal degeneration

In the discovery cohort $(n=186)$, a total of 206 different sequence alterations were recorded in the exons and intron-exon boundaries of USH2A; 88 variants were missense, coding insertions/deletions or splice site changes. Of these, 52 were rare with an MAF $<0.15 \%$ and thus were classified as likely disease-causing ( 21 were novel to this study and 31 were previously reported). Notably, 22 patients were found to harbour two of these likely disease-causing variants, whereas 24 were found to carry one likely disease-causing variant. In the latter group, one proband was found on MLPA testing to harbour a heterozygous duplication of exons 57-60. None of them was found 
to carry the c.7595-2144 A $>\mathrm{G}$ change, which causes inclusion of a pseudoexon. ${ }^{11}$

All 23 patients with two likely disease-causing variants were affected with adult-onset retinitis pigmentosa (Table 1). Therefore, $14 \%$ of patients with adult-onset recessive retinitis pigmentosa (23/168) were found to harbour two likely disease-causing variants in USH2A.

In the replication cohort of 84 patients with recessive retinal degeneration (syndromic and nonsyndromic), 25 additional probands with two likely disease-causing USH2A variants were identified. All 25 had a retinal phenotype consistent with retinitis pigmentosa; 11 of these had Usher syndrome type II and the remaining 14 reported no hearing complaint in childhood (Table 2).

When the third panel of 187 patients with nonsyndromic, adultonset, recessive retinitis pigmentosa was sequenced for exons 13, 51, 57 and 63, the following variants were identified: c.2276 G $>$ T (5 alleles); c.2299delG (3 alleles); c.2633 G > A, p.(Arg878His) (1 allele); c.10073 G > A, p.(Cys3358Tyr) (8 alleles); c.11156 G>A, p. (Arg3719Leu) (2 alleles); c.12575 G > A, p.(Arg4192His) (2 alleles); and c.12574C $>$ T, p.(Arg4192Cys) (1 allele). One subject harboured the c.2276 G > T variant in homozygous state, a second subject had c. $2276 \mathrm{G}>\mathrm{T}$ and c.12575 G > A, a third subject had biallelic c.2276$\mathrm{G}>\mathrm{T}$ and c.2299delG and two additional unrelated subjects carried the same pair of changes: c.2299delG and c.10073 G>A.

\section{Some USH2A alleles are only associated with nonsyndromic retinal disease}

We define as 'retinal disease-specific' variants or alleles that (i) were present in more than one patient with nonsyndromic retinal degeneration (in our discovery cohort and/or the literature) and (ii) have not been clearly associated with Usher syndrome type II to date (Table 3). On this basis, the following variants were categorised as likely 'retinal disease-specific': c.2802 T > G, p.(Cys934Trp); c.10073$\mathrm{G}>\mathrm{A}$; c.11156 G>A; c.12295-3 T>A; and c.12575 G>A. The c. $2276 \mathrm{G}>\mathrm{T}$ variant that has been previously associated mainly with disease confined to the eye $\mathrm{e}^{9,10}$ was also included in this group. Notably, the most prevalent c. $2276 \mathrm{G}>\mathrm{T}$ and c.10073 G > A variants were statistically significantly enriched in nonsyndromic cases compared with Usher syndrome type II cases $(P=0.0060$ and $P=0.047$, respectively (Fisher's exact test); the data on Usher syndrome type II were obtained from the UK National Collaborative Usher Study ${ }^{8}$ ). All 'retinal disease-specific' variants were located in laminin-type EGF-like domains or fibronectin type 3 domains (Figure 1).

On examining our data, it was rare for nonsyndromic cases to have two 'null' variants (ie, variants that are not missense and predicted to cause nonsense-mediated decay and/or significant truncation of the protein if translated), whereas this was common in those with Usher syndrome. Of 47 probands with nonsyndromic USH2A-related retinal degeneration (all three cohorts), only 5 had apparent biallelic 'null' variants (this is the exception due to c.12295-3 T $>$ C; see Table 1). This is significantly different to 39 out of 71 patients with USH2Aassociated Usher syndrome ${ }^{8}(P=0.0001$ (Fisher's exact test)). These data support the model that Usher syndrome represents the null phenotype consequent upon USH2A defects, and that 'retinal diseasespecific' alleles are partly functional, allowing them to contribute to normal cochlea development.

Assuming our model of allelic hierarchy is correct, further rare 'retinal disease-specific' alleles can be sought. In those probands with nonsyndromic retinal disease, an allele is likely to be 'retinal diseasespecific' if either (i) it is homozygous or (ii) it is paired with an allele that has previously and consistently been reported to be associated with Usher syndrome or (iii) it is paired with an allele that has previously and consistently been reported to be associated with nonsyndromic retinitis pigmentosa. On examining our discovery and replication cohorts in this way, the following alleles are likely to be 'retinal disease-specific': c.2332 G > T, p.(Asp778Tyr); c.3724C > T, p.(Pro1242Ser); c.4378 G>A, p.(Gly1460Arg); c.8546G $>$ T, p. (Gly2849Val); c.6904_6920dup17, p.(Gln2307Hisfs $\left.{ }^{\star} 25\right) ; \quad$ c.12580T > C, p.(Cys4194Arg) and c.15178 T>C, p.(Ser5060Pro). Further data from other cohorts of nonsyndromic patients are needed to confirm the 'retinal disease-specific' nature of these alleles.

\section{The c.12295-3T $>$ A, c.9056-2A $>$ G and c. c.5776+1G $>$ A variants result in abnormal USH $2 A$ pre-mRNA splicing}

The effect of c.12295-3 T > A, one of the probably 'retinal diseasespecific' changes, on splicing was investigated in subject D13. Primers were used to amplify a 2550 bp fragment between exons 58 and 64. PCR products of the expected size were amplified for the control sample, whereas a much smaller band was observed in the patient sample. Further cDNA analysis (Figure 2a) revealed the presence of an abnormal transcript (1033 bp) associated with an out-of-frame skipping of exon 63; this would result in a premature termination codon. Amplification of a $2371 \mathrm{bp}$ fragment between exons 62 and 66 combined with direct sequencing of the RT-PCR product confirmed the above findings. Notably, direct sequencing of the RT-PCR product demonstrated a normal sequence at position c.12093, where the sequencing of the genomic DNA identified a c.12093C > A, p. (Tyr4031*) change in heterozygous state. This suggests that the two likely disease-causing variants identified in this patient (c.12295-3 T > A and c.12093C > A) reside on two different alleles (Figure $2 \mathrm{~b}$ ). The non-amplification of the allele with c.12093C > A could be due to nonsense-mediated decay or preferential amplification of the smaller, exon-skipped PCR product from the other allele.

The effect of the c.9056-2 A $>$ G (likely 'retinal disease-specific') and c.5776+1 G > A (previously associated with Usher syndrome type II (LOVD-USHBase)) changes on splicing were investigated in subject D10; the c.9056-2 A $>$ G variant led to part of exon 46 being missed in the mRNA, while the c.5776+1 G>A variant was associated with skipping of exon 28. These results are summarised in Supplementary Figure S1.

\section{Defects in USH2A consistently cause retinitis pigmentosa}

The clinical features of 27 individuals with two likely disease-causing variants in USH2A (23 from discovery cohort plus 4 of their affected family members) are summarised in Table 1 . All 27 patients were noted to have typical features of retinitis pigmentosa such as pigmentary changes in the midperipheral retina and vessel attenuation. Patients usually presented with nyctalopia (median age of 24.5 years; range $12-42$ years). The median visual acuity at last visit was 0.24 $\log$ MAR (range -0.10 to 2.2 ). Six patients $(22 \%$ ) underwent cataract surgery at a median age of 47 (range 34-58) years. Central macular oedema was noted in 10 patients $(37 \%)$ at a median age of 50 (range 35-59) years.

\section{Fundus autofluorescence imaging is a clinically useful test for USH2A-related disease}

From the fundus autofluorescence images of 24 patients (48 eyes) three patterns were observed (Figure 3 ). Most patients ( $n=39$ eye; $81.3 \%$ ) showed preserved central autofluorescence surrounded by a variable diameter ring of high density ('hyperautofluorescent ring'; Figure 3, top row). Five (10.4\%) eyes had an abnormally increased signal in the fovea with no obvious hyperautofluorescent ring ('central 
Table 2 Genotype and clinical characteristics of patients with USH2A-related disease (replication cohort)

\begin{tabular}{|c|c|c|c|c|c|}
\hline \multirow[t]{2}{*}{ Subject } & \multicolumn{2}{|c|}{ USH2A sequencing results } & \multicolumn{2}{|c|}{ Likely effect of allele } & \multirow[t]{2}{*}{ Diagnosis } \\
\hline & Variant 1 & Variant 2 & Variant 1 & Variant 2 & \\
\hline R1 & c.2276G > T, p.(Cys759Phe) & c.2276G > T, p.(Cys759Phe) & Retina-specific & Retina-specific & Nonsyndromic retinitis pigmentosa \\
\hline R2 & c.2276G > T, p.(Cys759Phe) & c.1225T>C, p.(Trp409Arg) & Retina-specific & Unknown (novel) & Nonsyndromic retinitis pigmentosa \\
\hline R3 & c.2276G > T, p.(Cys759Phe) & c.9912dup, p.(Glu3305Argfs*41) & Retina-specific & Unknown & Nonsyndromic retinitis pigmentosa \\
\hline R4 & c.2276G > T, p.(Cys759Phe) & c.2299delG, p.(Glu767Serfs*21) & Retina-specific & Usher & Nonsyndromic retinitis pigmentosa \\
\hline R5 & c.99_100insT, (p.Arg34Serfs*41) & c.2802T $>$ G, p.(Cys934Trp) & Unknown & Retina-specific & Nonsyndromic retinitis pigmentosa \\
\hline R6 & c.5776G > A, p.(Glu1926Lys) & c.10073G > A, p.(Cys3358Tyr) & Usher & Retina-specific & Nonsyndromic retinitis pigmentosa \\
\hline R7 & c.5776G >A, p.(Glu1926Lys) & c. $10073 \mathrm{G}>\mathrm{A}, \mathrm{p} .($ Cys3358Tyr $)$ & Usher & Retina-specific & Nonsyndromic retinitis pigmentosa \\
\hline R8 & c.1256G > T, p.(Cys419Phe) & c. $11156 \mathrm{G}>\mathrm{A}$, p.(Arg3719His) & Usher & Retina-specific & Nonsyndromic retinitis pigmentosa \\
\hline R9 & c.1256G > T, p.(Cys419Phe) & c. $11156 \mathrm{G}>\mathrm{A}, \mathrm{p} .(\mathrm{Arg} 3719 \mathrm{His})$ & Usher & Retina-specific & Nonsyndromic retinitis pigmentosa \\
\hline R10 & c. $11864 G>A$, p. $\left(\operatorname{Trp} 3955^{*}\right)$ & c.12580T > C, p.(Cys4194Arg) & Usher & Unknown (novel) & Nonsyndromic retinitis pigmentosa \\
\hline R11 & c.8254G >A, p.(Gly2752Arg) & c. $15178 T$ > C, p.(Ser5060Pro) & Usher & Unknown (novel) & Nonsyndromic retinitis pigmentosa \\
\hline R12 & c.4378G > A, p.(Gly1460Arg) & c. $9424 G>T, p .($ Gly3142*) & Unknown (novel) & Usher & Nonsyndromic retinitis pigmentosa \\
\hline R13 & c.6904_6920dup17 & c.12877G > A, p.(Gly4293Ser) & Unknown (novel) & Unknown (novel) & Nonsyndromic retinitis pigmentosa \\
\hline R14 & c. 9611 A > G, p.(His3204Arg) & c.13768G > A, p.(Gly4590Ser) & Unknown (novel) & Unknown (novel) & Nonsyndromic retinitis pigmentosa \\
\hline R15 & c. $1876 \mathrm{C}>\mathrm{T}, \mathrm{p} .\left(\operatorname{Arg} 626^{*}\right)$ & c. $1876 \mathrm{C}>\mathrm{T}$, p. $\left(\operatorname{Arg} 626^{*}\right)$ & Usher & Usher & Usher syndrome \\
\hline $\mathrm{R} 16$ & c. $1256 \mathrm{G}>\mathrm{T}$, p.(Cys419Phe) & c.2299delG, p.(Glu767Serfs*21) & Usher & Usher & Usher syndrome \\
\hline R17 & c.2299delG, p.(Glu767Serfs*21) & c.14287G >A, p.(Gly4763Arg) & Usher & Usher & Usher syndrome \\
\hline R18 & c.2299delG, p.(Glu767Serfs*21) & c.14287G >A, p.(Gly4763Arg) & Usher & Usher & Usher syndrome \\
\hline R19 & c. $2209 \mathrm{C}>\mathrm{T}, \mathrm{p} .\left(\operatorname{Arg} 737^{*}\right)$ & c.2299delG, p.(Glu767Serfs*21) & Usher & Usher & Usher syndrome \\
\hline R20 & c.2299delG, p.(Glu767Serfs*21) & c. $5168-2 A>G$ & Usher & Unknown (novel) & Usher syndrome \\
\hline R21 & c.2299delG, p.(Glu767Serfs*21) & c. $5858-1 \mathrm{G}>\mathrm{A}$ & Usher & Unknown (novel) & Usher syndrome \\
\hline R22 & c.2299delG, p.(Glu767Serfs*21) & c.14180G > A, p. $\left(\operatorname{Trp} 4727^{\star}\right)$ & Usher & Unknown (novel) & Usher syndrome \\
\hline R23 & c.1679delC, p.(Pro560Leufs*31) & c. $11549-1 G>A$ & Unknown & Unknown (novel) & Usher syndrome \\
\hline R24 & c.854T > C, p.(Ile285Thr) & c.10724G > A, p.(Cys3575Tyr) & Unknown (novel) & Usher & Usher syndrome \\
\hline R25 & c. $2081 \mathrm{G}$ > C, p.(Cys694Ser) & c. 10612 C > T, p. $\left(\operatorname{Arg} 3538^{*}\right)$ & Unknown (novel) & Unknown (novel) & Usher syndrome \\
\hline
\end{tabular}

Retina-specific corresponds to likely 'retinal disease-specific' alleles (shown in bold).

Usher corresponds to likely 'Usher syndrome type II'-specific alleles.

Novel corresponds to alleles that are novel to this study. Of these novel changes, the following are found in the Exome Aggregation Consortium (ExAC) browser (accessed 31 December 2014): c.12580T $>$ C (1/122018), c.15178T $>$ C (4/122952 alleles), c.4378G >A (3/122140) and c.9611A > G (22/122680). Segregation analysis was performed in subjects R12, R20 and R22 and has confirmed that the reported variants are in trans.

Numbering of USH2A variants has been assigned in accordance with NCBI Reference Sequence NM_206933.2.

Table 3 Summary of phenotypes associated with the previously reported USH2A variants that were identified in the present series

\begin{tabular}{|c|c|c|c|c|c|c|c|}
\hline \multirow[t]{2}{*}{ Change in USH2A } & \multicolumn{6}{|c|}{ Number of previously reported cases } & \multirow[t]{2}{*}{ References } \\
\hline & $\begin{array}{c}\text { Nonsyndromic } \\
\text { retinitis pigmentosa }\end{array}$ & Usher type II & Atypical Usher & Usher. type I & Usher type III & Asymptomatic & \\
\hline c.2276G > T, p.(Cys759Phe) & 96 (12 hom) & 14 & 5 & $1^{\mathrm{a}}$ & - & 1 (hom) & $8,15-26$ \\
\hline c.2299delG, p.(Glu767Serfs*21) & 58 & 327 (46 hom) & 6 (3 hom) & - & 1 & - & $8-11,15-17,19-22,24,25,27-43$ \\
\hline c. $2332 \mathrm{G}>\mathrm{T}$ p.(Asp778Tyr) & - & 1 & - & - & - & - & 35 \\
\hline c.2802 T> G, p.(Cys934Trp) & 1 & - & - & - & - & - & 44 \\
\hline c.3902G > T, p.(Gly1301Val) & - & - & - & $1^{*}$ & - & - & 40 \\
\hline c. $5776+1 G>A$ & - & 4 & - & - & 1 & - & $21,24,25,40$ \\
\hline 7595-3C > G, p.Pro2533Asnfs*5 & - & 5 & - & - & - & - & $8,20,45$ \\
\hline c. $9371+1 G>C$ & - & 1 & - & - & - & - & 8 \\
\hline c. 10073 G > A, p.(Cys3358Tyr) & 5 & - & 1 & - & - & - & $5,8,23,26,41$ \\
\hline c. $11156 \mathrm{G}>\mathrm{A}$, p.(Arg3719His) & 1 & - & - & - & - & - & 5 \\
\hline c. $12295-3 \mathrm{~T}>\mathrm{A}$ & - & - & $1^{\mathrm{b}}$ & - & - & - & 8 \\
\hline c. 12575 G > A, p.(Arg4192His) & 4 (1 hom) & - & $1^{\mathrm{c}}$ (hom) & - & - & - & $5,8,23,26$ \\
\hline c. $13010 \mathrm{C}>\mathrm{T}, \mathrm{p} .($ Thr4337Met $)$ & - & 2 & - & - & - & - & 5,35 \\
\hline c. $13316 \mathrm{C}>\mathrm{T}, \mathrm{p} .($ Thr4439Ile) & - & 5 & - & - & - & - & $8,21,46$ \\
\hline c. $14426 \mathrm{C}>\mathrm{T}$ p.(Thr4809|le) & - & 3 & - & - & - & - & 8,36 \\
\hline
\end{tabular}

Numbering of USH2A variants has been assigned in accordance with NCBI Reference Sequence NM_206933.2. The complete list of references can be found in LOVD-USHBase.

${ }^{a}$ A single heterozygous variant in MYO7A was also reported in this patient; *a homozygous (hom) variant in MYO7A was also reported in this patient.

bAfter reviewing the clinical data, the patient was categorised as atypical due to adult onset of hearing loss (45 years old) and normal speech.

${ }^{c}$ After reviewing the clinical data, the patient was categorised as atypical due to very mild, progressive hearing loss. 
hyperautofluorescence'; Figure 3, middle row). Four (8.3\%) eyes were characterised by widespread hypoautofluorescence corresponding to retinal pigment epithelial atrophy ('severely decreased autofluorescence'; Figure 3, bottom row). The findings were concordant between the eyes in all but two patients. Overlaying of fundus autofluorescence and OCT images suggested that the

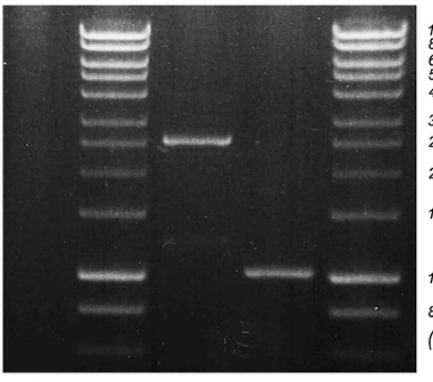

NTC

Control Subject D13

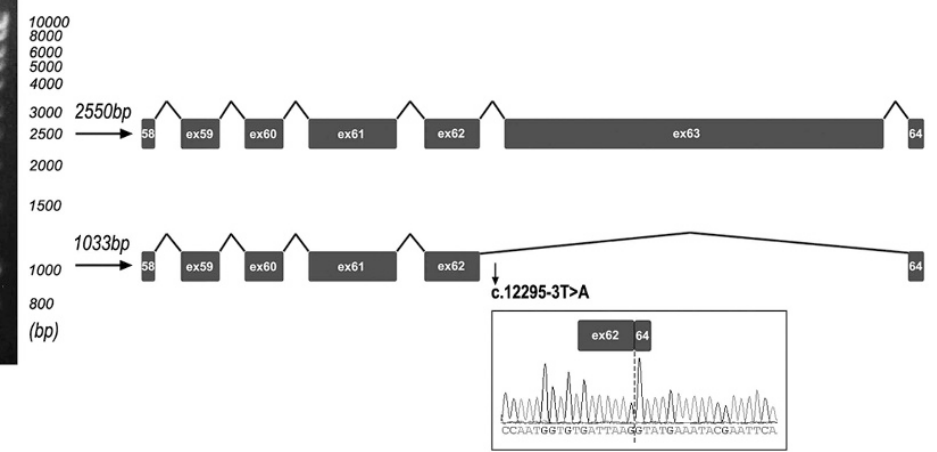

\section{b}

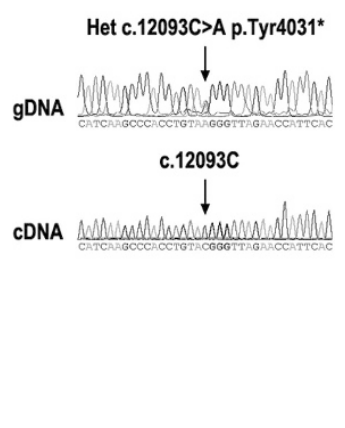

Figure 2 (a) RT-PCR analysis of the c.12295-3T>A mutation. RT-PCR was performed on RNA extracted from nasal epithelial cells of subject D13 and an unrelated control individual, using primers located in exons 58 and 64 of USH2A. In subject D13, RT-PCR produced a shorter product of 1033 bp corresponding to skipping of USH2A exon 63 (partial sequence chromatogram of this transcript is shown in the box; the dashed line indicates the splice junction between exons 62 and 64). The other allele of subject D13 harbouring a c.12093C>A, p.(Tyr4031*) mutation did not amplify. Amplification on control template produced a band of $2550 \mathrm{bp}$, corresponding to wild-type sequence. (b) Partial sequence chromatogram of genomic DNA from subject D13 showing a heterozygous c.12093C > A variant in exon 62. Sequence analysis of the corresponding RT-PCR product (1033 bp, see above) revealed a normal sequence at c.12093; this implies that the c.12295-3T > A and c.12093C > A variants reside on different alleles. NTC stands for no template control.

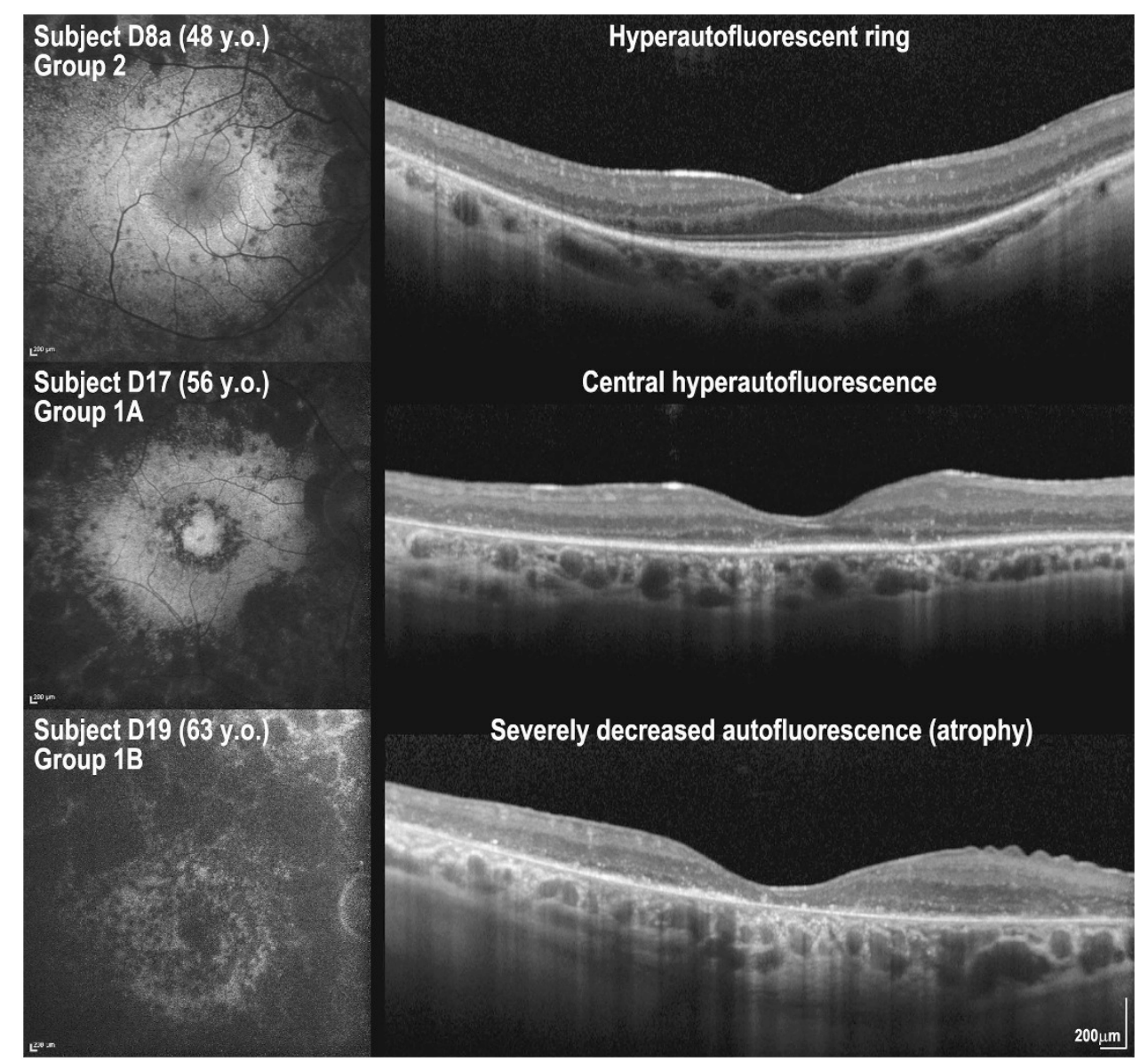

Figure 3 Fundus autofluorescence (FAF) imaging and foveal OCT scans of three patients with USH2A retinopathy. Three patterns were observed: (i) a hyperautofluorescent ring on FAF and preserved photoreceptor inner segment ellipsoid line in the area within the hyperautofluorescent ring on OCT (subject D8a; top row); (ii) central hyperautofluorescence on FAF and absent photoreceptor inner segment ellipsoid line on OCT (subject D17; middle row), and (iii) severely decreased autofluorescence on FAF and absent outer retina layers with thinning of the retinal pigment epithelium/Bruch's membrane complex band (subject D19; bottom row). Y.o., years old. 
hyperautofluorescent ring represents a border between relatively preserved and diseased retinal tissue (Figure 3), and future structure-function correlation studies are expected to provide important insights into the clinical utility of this imaging modality

\section{Audiological phenotype in $U S H 2 A$ retinopathy is variable}

The 23 patients with two likely disease-causing variants from the discovery cohort and their four affected relatives reported no hearing loss in childhood, with 9 (33\%) of these reporting subjective adultonset hearing loss. Audiological assessment was conducted in 19 of these patients. In $14(74 \%)$ patients, it was consistent with a Group 1 phenotype (ie, thresholds within normal limits); of those, $9(47 \%)$ were classified in Group 1A and 5 (26\%) in Group 1B. Three (16\%) patients were classified in Group 2 and $2(10 \%)$ in Group 3 (Supplementary Figure S2). Qualitative analysis revealed the c.12295$3 \mathrm{~T}>\mathrm{A}$ variant to be correlated with a more severe audiological phenotype (Groups 2 and 3). There seems to be no obvious correlation between the severity of visual and audiological phenotypes (Table 1, Figure 4 and Supplementary Figure S2).

\section{DISCUSSION}

In the present study, we confirm that recessive variants affecting USH2A function are a common cause of retinitis pigmentosa with disease-causing variants being spread throughout the gene. When allelic heterogeneity was studied and compared with that reported in
Usher syndrome, the concept of 'retinal disease-specific' USH2A alleles (ie, alleles associated with retinal degeneration and no hearing complaint in childhood) became apparent. The presence of at least one such allele in a patient with USH2A-related retinal degeneration results in relative preservation of hearing (Figure 5). Five likely 'retinal disease-specific' variants (c.2802T $>$ G; c.10073G $>$ A; c.11156G $>$ A; c.12295-3T $>$ A and c.12575G $>$ A) that are novel to this study were identified in addition to c.2276G $>\mathrm{T}$, a relatively common sequence alteration previously associated with retinitis pigmentosa without hearing impairment. ${ }^{9,10}$

Defects in the USH2A gene have been previously reported to account for $12-25 \%$ of all retinitis pigmentosa cases, dominant, recessive or X-linked; syndromic or nonsyndromic. ${ }^{5}$ McGee et al ${ }^{5}$ reported that among 80 patients with nonsyndromic recessive retinitis pigmentosa, $23 \%$ had one or two likely disease-causing variants in USH2A. This result was consistent with our findings: $24.7 \%(46 / 186)$ of patients with recessive retinal degeneration and no reported hearing loss in childhood harboured one or two likely disease-causing changes. Many variants in USH2A can be overlooked when only coding regions and intron-exon boundaries are sequenced. In previous studies, it was found that screening for duplications, deletions and a common deep-intronic sequence alteration (c.7595-2144A $>$ G) detected a second disease-causing variant in $35 \%$ of cases with Usher syndrome type II that had only one variant affecting function on conventional Sanger sequencing of all USH2A exons. ${ }^{11,47}$ In the present cohort,

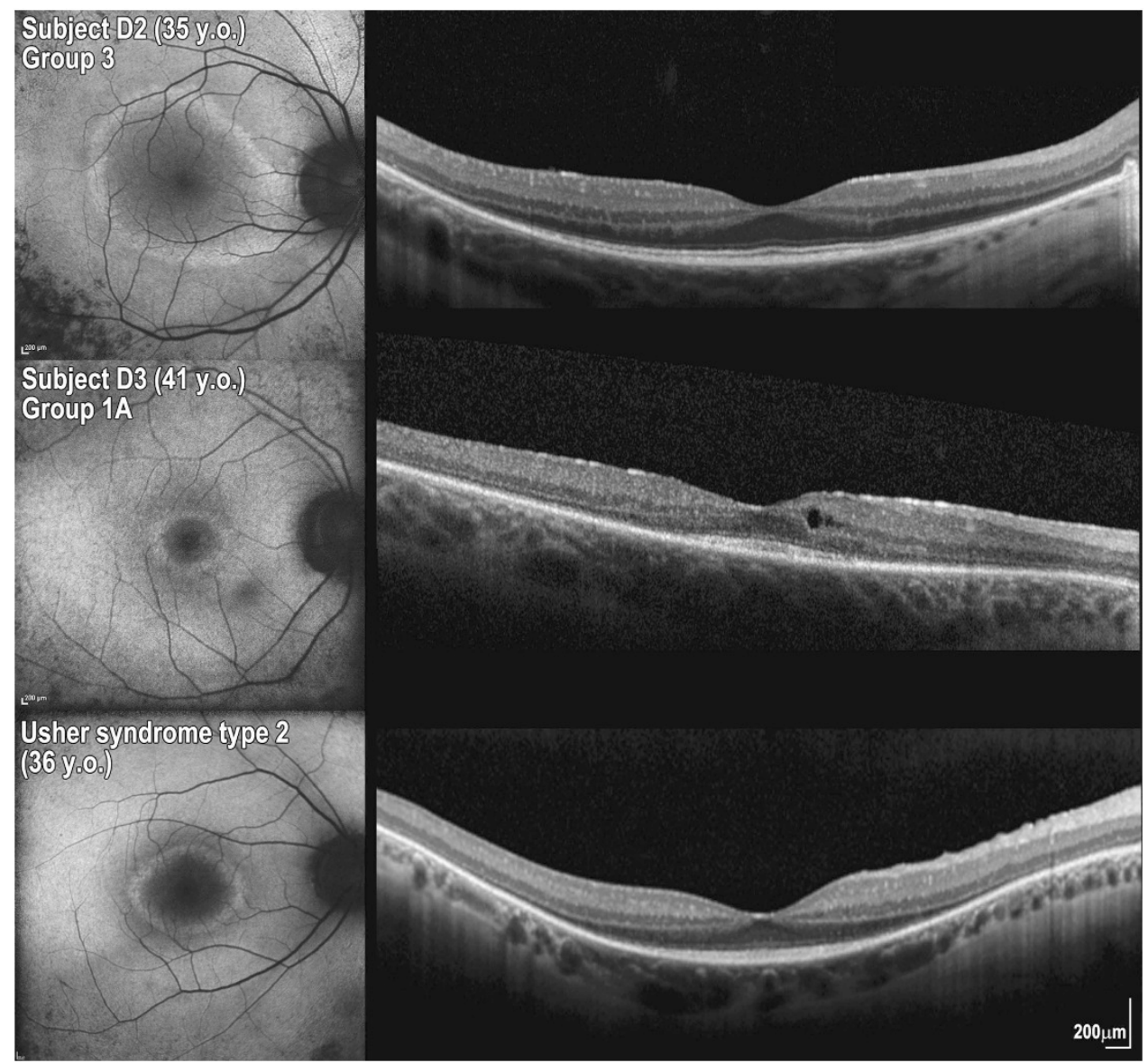

Figure 4 Variability in the severity of ocular and audiological phenotype due to mutations in USH2A in three patients of similar age. Fundus autofluorescence (FAF) imaging and foveal OCT show a better preserved retina in subject D2 (group 3 corresponding to abnormal audiological assessment) and in a patient with Usher syndrome type II compared with subject D3 (middle panel), who has normal hearing. 

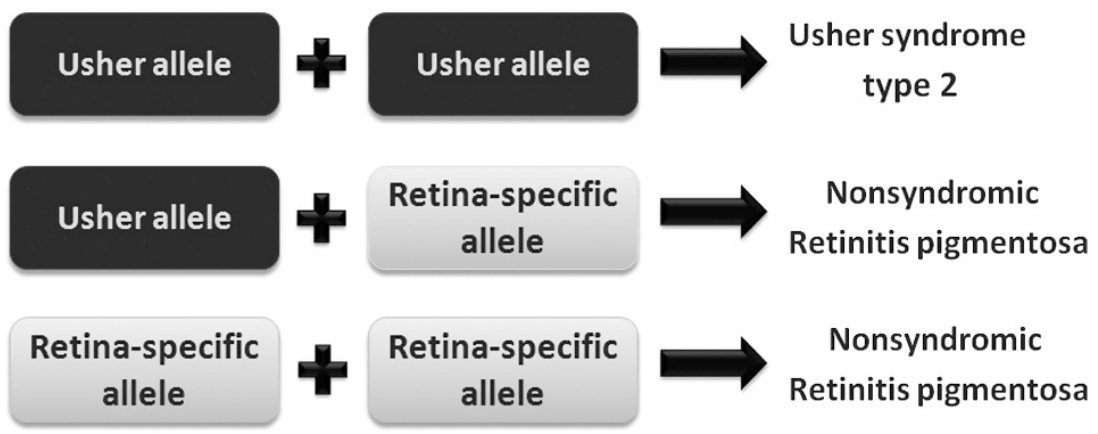

Figure 5 Schematic showing the proposed allelic hierarchy of USH2A mutations: the presence of at least one retinal disease-specific ('retina-specific') USH2A allele in a patient with USH2A-related disease results in normal hearing at least in childhood.

despite performing MLPA analysis and testing for the c.7595$2144 \mathrm{~A}>\mathrm{G}$ change, a significant number of cases with only one likely disease-causing in USH $2 A$ variant remained $(23 / 186,12.4 \%)$. This observation can be attributed to (i) a number of variants affecting function still being overlooked and/or (ii) some changes defined here as likely disease-causing being rare benign polymorphisms.

The first report of an USH2A change being associated with recessive retinitis pigmentosa without hearing impairment was by Rivolta et al 9 in 2000; this change was a $G$ to $T$ transversion in exon 13 (c.2276G $>$ T).${ }^{9}$ Since then many studies have confirmed this finding (Table 1) and the c.2276G $>\mathrm{T}$ variant is often considered to be the most common disease-causing variant in patients with nonsyndromic retinitis pigmentosa. ${ }^{9,10,24}$ In the present study, c.2276G $>$ T was found in $6.5 \%(12 / 186)$ of cases; in all study subjects, it was in cis with a previously reported polymorphism c.2256T $>$ C, p.(His752His), suggesting a common ancestral haplotype. However, previous reports have shown that the c.2276G $>\mathrm{T}$ can be a recurrent sequence alteration. ${ }^{17}$ We have found five additional changes that are also associated with retinitis pigmentosa and no hearing complaint in childhood (presumed 'retinal disease- specific' variants). These include four previously reported missense variants (c.10073G $>A$, , ,8,23,26,41 c. $2802 \mathrm{~T}>\mathrm{G},{ }^{44}$ c. $11156 \mathrm{G}>\mathrm{A}^{5}$ and c. $\left.12575 \mathrm{G}>\mathrm{A}^{5,8,23,26,41}\right)$ and a splice site change (c.12295-3T $>A^{8}$ ). We have shown that the latter results in exclusion of exon 63 and an out-of-frame deletion. Three out of four patients harbouring this variant had no auditory complaint, despite the presence of an abnormal auditory phenotype, which would suggest a long-standing or very slowly progressive neurosensory hearing impairment (Table 1).

Analysis of allelic heterogeneity in $U S H 2 A$ in our discovery cohort (23 probands with presumed USH2A-related retinitis pigmentosa and no hearing complaint in childhood) revealed that all but one (patient D9) patient harboured at least one 'retinal disease-specific' or novel (presumed 'retinal disease-specific') USH2A allele (Table 1). Importantly, a similar pattern (Figure 5) was observed in our replication cohort (16 probands with presumed USH2A-related retinitis pigmentosa and no hearing complaint in childhood; Table 3). Only in one case (patient D9; Table 1) there appeared to be discordance: a c.2332G > T change was identified in homozygous state in a Somali patient with retinitis pigmentosa and normal audiometric testing; previously, this change has been reported in heterozygous state in a patient with Usher syndrome type $\mathrm{II}^{35}$ One explanation for this could be that this is a rare polymorphism. Recently, a similar allelic hierarchy has been reported for change affecting $\mathrm{CDH} 23$ function: a 'nonsyndromic deafness' $\mathrm{CDH} 23$ allele in trans configuration with a 'syndromic/Usher syndrome type I' $\mathrm{CDH} 23$ allele preserves vision and balance in deaf individuals. ${ }^{48}$
Audiometric findings were within normal limits for most patients with two likely disease-causing USH2A variants and no hearing complaint in childhood (14 of 19 tested; Table 1). Notably, the severity of the retinal phenotype did not obviously correlate with the severity of the hearing impairment (Figure 4); this is in keeping with previous reports. ${ }^{24}$ Interestingly, the eldest subject D23 in the present study reported adult-onset hearing loss and had a hearing defect consistent with Usher syndrome type II at age 75 years. This is in keeping with the notion that recessive variants in USH2A cause a spectrum of hearing defects that range from an early-onset phenotype consistent with Usher syndrome type II to completely normal hearing.

We have shown that an allelic hierarchy of variants affecting USH2A function is likely with 'retinal disease-specific' alleles being phenotypically dominant to 'Usher syndrome type II' alleles. Although this finding has implications for counselling, the fact that USH2A diseasecausing variants are often private makes prediction of the fully evolved phenotype challenging. In any case, the audiological phenotype in USH2A-related disease is highly variable and a multidisciplinary approach is often relevant even to cases without hearing complaints in childhood.

\section{CONFLICT OF INTEREST}

The authors declare no conflict of interest.

\section{ACKNOWLEDGEMENTS}

We acknowledge the help provided by the family members in this study and the colleagues who referred patients to us. The work was partially funded by the National Institute for Health Research UK (Moorfields Eye Hospital and Institute of Ophthalmology Biomedical Research Centre; Great Ormond Street Hospital and Institute of Child Health Biomedical Research Centre; University College London Hospitals NHS Foundation Trust and University College London Biomedical Research Centre), RP Fighting Blindness (formerly British Retinitis Pigmentosa Society) and the Wellcome Trust (Grant No. 098051).

\footnotetext{
1 Buch H, Vinding T, La Cour M, Appleyard M, Jensen GB, Nielsen NV: Prevalence and causes of visual impairment and blindness among 9980 Scandinavian adults: the Copenhagen City Eye Study. Ophthalmology 2004; 111: 53-61.

2 Wright AF, Chakarova CF, Abd El-Aziz MM, Bhattacharya SS: Photoreceptor degeneration: genetic and mechanistic dissection of a complex trait. Nat Rev Genet 2010; 11: 273-284

3 den Hollander Al, Black A, Bennett J, Cremers FP: Lighting a candle in the dark advances in genetics and gene therapy of recessive retinal dystrophies. J Clin Invest 2010: 120: 3042-3053.

4 Hartong DT, Berson EL, Dryja TP: Retinitis pigmentosa. Lancet 2006; 368: 1795-1809.

5 McGee TL, Seyedahmadi BJ, Sweeney MO, Dryja TP, Berson EL: Nove mutations in the long isoform of the USH2A gene in patients with Usher syndrome type II or non-syndromic retinitis pigmentosa. J Med Genet 2010; 47 499-506.
} 
6 van Wijk E, Pennings RJ, te Brinke $\mathrm{H}$, et al: Identification of 51 novel exons of the Usher syndrome type 2A (USH2A) gene that encode multiple conserved functional domains and that are mutated in patients with Usher syndrome type II. Am J Hum Genet 2004;74:738-744.

7 Liu X, Bulgakov OV, Darrow KN, et al: Usherin is required for maintenance of retinal photoreceptors and normal development of cochlear hair cells. Proc Natl Acad Sci USA 2007; 104:4413-4418.

8 Le Quesne Stabej P, Saihan Z, Rangesh N, et al: Comprehensive sequence analysis of nine Usher syndrome genes in the UK National Collaborative Usher Study. J Med Genet 2012;49:27-36.

9 Rivolta C, Sweklo EA, Berson EL, Dryja TP: Missense mutation in the USH2A gene: association with recessive retinitis pigmentosa without hearing loss. Am J Hum Genet 2000; 66: 1975-1978.

10 Bernal S, Ayuso C, Antinolo G, et al: Mutations in USH2A in Spanish patients with autosomal recessive retinitis pigmentosa: high prevalence and phenotypic variation. J Med Genet 2003;40:e8.

11 Vache $C$, Besnard T, le Berre $P$, et al: Usher syndrome type 2 caused by activation of an USH2A pseudoexon: implications for diagnosis and therapy. Hum Mutat 2012;33: 104-108.

12 British Society of Audiology: Recommended Procedure: Pure-Tone Air-conduction and Bone-Condiction Threshold Audiometry With and Without Masking. British Society of Audiology, Reading, Berkshire, UK, 2011.

13 Davis AC: Hearing in Adults. London, Whurr Publishers Ltd, 1995.

14 Parving A, Newton V: Editorial guidelines for description of inherited hearing loss. J Audiol Med 1995; 4: ii-i.

15 Dreyer B, Tranebjaerg L, Rosenberg T, Weston MD, Kimberling WJ, Nilssen O: Identification of novel USH2A mutations: implications for the structure of USH2A protein. Eur J Hum Genet 2000; 8: 500-506.

16 Nájera C, Beneyto M, Blanca J et al: Mutations in myosin VIIA (MYO7A) and usherin (USH2A) in Spanish patients with Usher syndrome types I and II, respectively. Hum Mutat 2002; 20: 76-77.

17 Aller E, Najera C, Millan JM et al: Genetic analysis of 2299delG and C759F mutations (USH2A) in patients with visual and/or auditory impairments. Eur J Hum Genet 2004; 12: 407-410.

18 Seyedahmadi BJ, Rivolta C, Keene JA, Berson EL, Dryja TP: Comprehensive screening of the USH2A gene in Usher syndrome type II and non-syndromic recessive retinitis pigmentosa. Exp Eye Res 2004; 79: 167-173.

19 Bernal S, Medà C, Solans T et al: Clinical and genetic studies in Spanish patients with Usher syndrome type II: description of new mutations and evidence for a lack of genotype-phenotype correlation. Clin Genet 2005; 68: 204-214.

20 Baux D, Larrieu L, Blanchet $C$ et al: Molecular and in silico analyses of the full-length isoform of usherin identify new pathogenic alleles in Usher type II patients. Hum Mutat 2007; 28: 781-789.

21 Dreyer B, Brox V, Tranebjaerg L et al: Spectrum of USH2A mutations in Scandinavian patients with Usher syndrome type II. Hum Mutat 2008; 29: 451

22 Herrera W, Aleman TS, Cideciyan AV et al: Retinal disease in Usher syndrome III caused by mutations in the clarin-1 gene. Invest Ophthalmol Vis Sci 2008; 49: 2651-2660.

23 Avila-Fernandez A, Cantalapiedra D, Aller E et al: Mutation analysis of 272 Spanish families affected by autosomal recessive retinitis pigmentosa using a genotyping microarray. Mol Vis 2010; 16: 2550-2558.

24 Sandberg MA, Rosner B, Weigel-DiFranco C, McGee TL, Dryja TP, Berson EL: Disease course in patients with autosomal recessive retinitis pigmentosa due to the USH2A gene. Invest Ophthalmol Vis Sci 2008; 49: 5532-5539.

25 Jaijo T, Aller E, García-García G et al: Microarray-based mutation analysis of 183 Spanish families with Usher syndrome. Invest Ophthalmol Vis Sci 2010; 51: 1311-1317.

26 Neveling K, Collin RW, Gilissen C et al: Next-generation genetic testing for retinitis pigmentosa. Hum Mutat 2012; 33: 963-972.

27 Eudy JD, Weston MD, Yao S et al: Mutation of a gene encoding a protein with extracellular matrix motifs in Usher syndrome type Ila. Science 1998; 280: 1753-1757.

28 Liu XZ, Hope C, Liang CY et al: A mutation (2314delG) in the Usher syndrome type IIA gene: high prevalence and phenotypic variation. Am J Hum Genet 1999; 64: 1221-1225.

29 Weston MD, Eudy JD, Fujita S et al: Genomic structure and identification of nove mutations in usherin, the gene responsible for Usher syndrome type Ila. Am J Hum Genet 2000; 66: 1199-1210.
30 Leroy BP, Aragon-Martin JA, Weston MD et al: Spectrum of mutations in USH2A in British patients with Usher syndrome type II. Exp Eye Res 2001; 72: 503-509.

31 Ouyang XM, Hejtmancik JF, Jacobson SG et al: Mutational spectrum in Usher syndrome type II. Clin Genet 2004; 65: 288-293.

32 Pennings RJ, Huygen PL, Orten DJ et al: Evaluation of visual impairment in Usher syndrome $1 \mathrm{~b}$ and Usher syndrome 2a. Acta Ophthalmol Scand 2004; 82: 131-139.

33 Pennings RJ, Te Brinke $\mathrm{H}$, Weston MD et al: USH2A mutation analysis in 70 Dutch families with Usher syndrome type II. Hum Mutat 2004; 24: 185.

34 Schwartz SB, Aleman TS, Cideciyan AV et al: Disease expression in Usher syndrome caused by VLGR1 gene mutation (USH2C) and comparison with USH2A phenotype. Invest Ophthalmol Vis Sci 2005; 46: 734-743.

35 Aller E, Jaijo T, Beneyto $\mathrm{M}$ et al: Identification of 14 novel mutations in the long isoform of USH2A in Spanish patients with Usher syndrome type II. J Med Genet 2006; 43: e55.

36 Ebermann I, Koenekoop RK, Lopez I, Bou-Khzam L, Pigeon R, Bolz HJ: An USH2A founder mutation is the major cause of Usher syndrome type 2 in Canadians of French origin and confirms common roots of Quebecois and Acadians. Eur J Hum Genet 2009; 17: 80-84.

37 Leijendeckers JM, Pennings RJ, Snik AF, Bosman AJ, Cremers CW: Audiometric characteristics of USH2a patients. Audiol Neurootol 2009; 14: 223-231.

38 Yan D, Ouyang X, Patterson DM, Du LL, Jacobson SG, Liu XZ: Mutation analysis in the long isoform of USH2A in American patients with Usher Syndrome type II. J Hum Genet 2009; 54: 732-738.

39 Kimberling WJ, Hildebrand MS, Shearer AE et al: Frequency of Usher syndrome in two pediatric populations: Implications for genetic screening of deaf and hard of hearing children. Genet Med 2010; 12: 512-516.

40 Bonnet C, Grati M, Marlin S et al: Complete exon sequencing of all known Usher syndrome genes greatly improves molecular diagnosis. Orphanet J Rare Dis 2011; 6: 21.

41 Garcia-Garcia G, Aparisi MJ, Jaijo T et al: Mutational screening of the USH2A gene in Spanish USH patients reveals 23 novel pathogenic mutations. Orphanet J Rare Dis 2011; 6: 65

42 Vozzi D, Aaspollu A, Athanasakis E et al: Molecular epidemiology of Usher syndrome in Italy. Mol Vis 2011; 17: 1662-1668.

43 Domanico D, Fragiotta S, Trabucco P, Nebbioso M, Vingolo EM: Genetic analysis for two italian siblings with usher syndrome and schizophrenia. Case Rep Ophthalmol Med 2012; 2012: 380863.

44 Xu W, Dai H, Lu T, Zhang X, Dong B, Li Y: Seven novel mutations in the long isoform of the USH2A gene in Chinese families with nonsyndromic retinitis pigmentosa and Usher syndrome Type II. Mol Vis 2011; 17: 1537-1552.

45 Le Guédard-Méreuze S, Vaché C, Baux D et al: Ex vivo splicing assays of mutations at noncanonical positions of splice sites in USHER genes. Hum Mutat 2010; 31 347-355.

46 Ebermann I, Phillips JB, Liebau MC et al: PDZD7 is a modifier of retinal disease and a contributor to digenic Usher syndrome. J Clin Invest 2010; 120: 1812-1823.

47 Steele-Stallard HB, Le Quesne Stabej P, Lenassi E et al: Screening for duplications, deletions and a common intronic mutation detects $35 \%$ of second mutations in patients with USH2A monoallelic mutations on Sanger sequencing. Orphanet $J$ Rare Dis 2013; 8: 122

48 Schultz JM, Bhatti R, Madeo AC et al: Allelic hierarchy of $\mathrm{CDH} 23$ mutations causing non-syndromic deafness DFNB12 or Usher syndrome USH1D in compound heterozygotes. J Med Genet 2011; 48: 767-775.

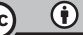

This work is licensed under a Creative Commons Attribution 3.0 Unported License. The images or other third party material in this article are included in the article's Creative Commons license, unless indicated otherwise in the credit line; if the material is not included under the Creative Commons license, users will need to obtain permission from the license holder to reproduce the material. To view a copy of this license, visit http:// creativecommons.org/licenses/by/3.0/

Supplementary Information accompanies this paper on European Journal of Human Genetics website (http://www.nature.com/ejhg) 